\title{
Thermal behavior of concentrated oil-in-water emulsions based on soybean oil and palm kernel olein blends
}

\begin{abstract}
Droplet size distribution and thermal behavior of concentrated oil-in-water emulsions based on soybean oil (SBO)/palm kernel olein (PKO) blends were investigated. The emulsions were prepared using $70 \%$ (wt./wt.) oil blends of SBO/PKO as dispersed phases and stabilized by egg yolk. An increase in PKO level (0-40\% wt./wt.) in the oil dispersed phase volume fraction caused significant increases $(\mathrm{p}<0.05)$ in volume-weighted mean diameter $(\mathrm{d} 4,3)$. The DSC data suggested that crystallization of the emulsions was induced by a 'template effect' of yolk constituents via a surface heterogeneous nucleation. Emulsions with 0-20\% (wt./wt.) PKO levels in the dispersed phase demonstrated a good cool-heat stability even after three successive thermal cycles (from $50{ }^{\circ} \mathrm{C}$ to $-70{ }^{\circ} \mathrm{C}$ at $10 \mathrm{~min} /{ }^{\circ} \mathrm{C}$ ). After the first thermal cycle, emulsions with $30 \%$ and $40 \%$ PKO levels in the oil dispersed phase were destabilized due to strong coalescence and crystallized via volume-surface heterogeneous nucleation. The unstable emulsions were attributable to high level of saturated triacylglycerols from $\mathrm{PKO}$, with high droplet size characteristic, causing them to be more prone to partial coalescence.
\end{abstract}

Keyword: Soybean oil; Palm kernel olein; Droplet size; DSC; Heterogeneous nucleation; Emulsion stability; Coalescence 